be applied to substances such as glucose or amino-acids. Some general impairment of absorption was suggested by our finding that in the active stages of sprue the dry weight of the stools was increased to a greater extent than could be accounted for by their increased fat content. Using indirect methods, we confirmed that glucose absorption is often impaired, and we also found some evidence of faulty absorption of iron, sodium, and chloride in certain cases. On the other hand, iodide and amino-acids were abnormally absorbed. Iodine is absorbed from the stomach and so is rather a special case.

The satisfactory absorption of amino-acids does show, however, that the intestinal absorption defect is not universal; the exact process involved in amino-acid absorption is not known, but absorption is more rapid than can be accounted for by simple diffusion (Höber, 1945). Fourman (1947) has found evidence of impaired xylose absorption in sprue: this may imply a more fundamental absorption defect than that shown by flat glucose curves, since xylose absorption, unlike that of glucose, is not inhibited by phloridzin and may therefore be produced by 9 different and simpler mechanism.

In summary, the evidence suggests that there is in fact a fairly general impaired absorption in sprue, and that fat malabsorption has been first observed only because its effects on the stools are more obvious.

\section{Salt Deficiency}

In preliminary work on the syndrome described above as "severe sprue" it was found that the serum sodium and chloride were both low. A balance experiment showed that large amounts of sodium and chloride were being lost in the watery stools, and that sodium was practically absent from the urine although chloride was still present in about half the normal amount. This is the biochemical pattern of salt deficiency (Black, 1946) accompanied by some acidosis ; the absence of sodium from the urine is very much against suprarenal insufficiency being the cause of the hypotension-a suggestion made by Thaysen (1932). The persistence of some chloride in the urine may be misleading if reliance is placed on the urinary chloride alone in detecting salt deficiency in this type of patient.

\section{Conclusion}

We would emphasize the two findings which seem to us of practical therapeutic significance. The first is that yeast extract in large doses has a favourable effect on fat absorption: we have seen clinical benefit from yeast treatment in patients with chronic sprue in this country, and it is more convenient for the patient than frequent injections of crude liver extract. Secondly, a number of patients with sprue became acutely. ill, with salt-deficiency dehydration associated with watery diarrhoea ; in such patients the diarrhoea must be controlled with sulphaguanidine and parenteral liver, and their diet should also be supplemented with moderate amounts of salt.

\section{REFERENCES}

Black, D. A. K. (1946), Lancet, 2, 671.

Bound, J. P., and Fourman, L. P. R. (1947). Quart. J. Med. In the press.

and Simpson, J. A. (1947). Proc. Soc. trop. Med. Hyg. In the press.

Bloor, W. R. (1943). Biochemistry of the Fatty Acids. New York. Castle, $\mathbf{W}$. B. Rhoads, C. P. Lawson, H. A., and Payne, G. C. (1935). Arch. intern. Med., 56, 627

Cooke, W. T., Elkes, J. J., Frazer, A. C., Parkes, J., Peeney, A. I. P., Sammons, H. G., and Thomas, G. (1946). Quart. J. Med., 15,141 .

Davidson, L. S. P., Girdwood, R. H., and Innes, E. M. (1947). Lancet, 1,511 .

Fourman, L. P. R. (1947). To be published.

Frazer, A. C. (1946). Physiol. Rev., 26, 103.

Hëber, R. (1945). Physical Chemistry of Cells and Tissues. London. Thaysen, T. E. H. (1932). Non-tropical Sprue. London.

\section{SHOCK IN OBSTETRICS}

BY

\author{
W. A. SCOTT, F.R.C.S.Ed., F.R.C.O.G. \\ Professor Emeritus in Obstetrics and Gynaecology, \\ University of Toronto
}

The essential mechanism of surgical shock is yet to be definitely established. It is understandable, therefore, that the physiologists and experimental surgeons desire to keep surgical shock in a special category until the mechanism of its inception may be discovered. As regards this subject I am neither a physiologist nor an experimental surgeon, and I speak, therefore, simply as a clinician. To the clinician there is a group of cases characterized by circulatory deficiency, with low blood pressure, decreased blood volume, decreased cardiac output, and increased concentration of blood that present a common clinical picture irrespective of the primary cause. These patients present a group of symptoms and not a disease. The prevention and treatment of these are of primary importance, and whether there is a special kind of shock called " obstetric shock" occurring in normal women after normal labour is of little importance. At one time it was customary to attribute a certain number of sudden deaths during or after labour to primary shock; but Sheehan, as well as other observers, has made it quite clear that in' the great majority, if not all, of these cases necropsy will reveal some condition sufficient to account for the fatality.

In all urban centres the number of maternal deaths certified as due to "shock" is a great deal less than it was twenty-five years ago. Of 122 maternal deaths during a two-year period from May, 1938, carefully evaluated by the Department of Health and Welfare of the Province ot Manitoba, five were originally attributed to "strain and shock." Two of these, on review of the clinical history", were obviously due to internal haemorrhage, and the other three, on review, could not easily be attributed to shock. All five of these patients were delivered at home, remote from hospitals, and necropsies were not done.

Table $I$ is an analysis of the maternal deaths with clinical evidences of shock in the Province of Ontario for 1943, the last year for which complete data were available.

TABLE I.-Maternal Deaths Accompanied by Shock: Province of Ontario, 1943

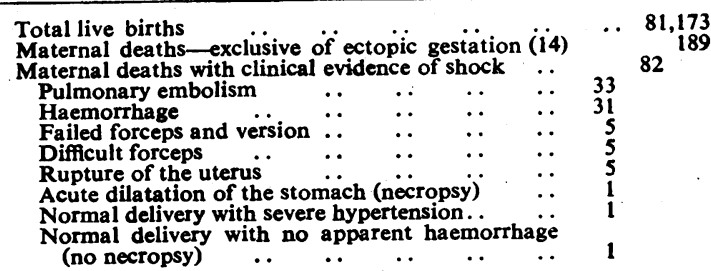

It will be seen that at the most only 11 of these did not have definite lesions accounting for the shock, and of the 11 there were 5 versions after failed forceps

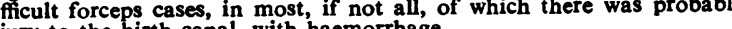
severe injury to the birth canal, with haemorrhage.

It is my opinion that, from the clinical point of view, the great majority of cases of obstetric shock can be evaluated in terms of haemorrhage. That is not to say that other causes of shock do not occur in the parturient patient : it is to say that they are relatively infrequent.

Table II summarizes the cases of shock on the public ward service of the Toronto General Hospital for the period $1931-43$.

In the third group, with a-blood loss of more than $1,000 \mathrm{ml}$., it will be noted that there were 6 deaths. Two of these were cases of placenta praevia; both had severe haemorrhages before admission and were bleeding when 
admitted. Both cases had immediate transfusion and were treated by version. I would like to point out that this method of treatment is rarely employed in our service, and that there were particular circumstances which seemed to warrant it in these instances ; but probably both cases were errors of judgment. One of these patients died following post-partum haemorrhage. The second patient recovered from her shock, had a spontaneous delivery but a post-partum haemorrhage, and died in two hours in spite of further transfusion.

TABLE II.-Toronto General Hospital

Total deliveries, 12,749

126 cases with blood loss $500-700 \mathrm{ml}$. and/or signs of mild shock Recovered within 15 minutes

2 cases with mild shock and minimum blood loss but difficult deliveries

64 cases with blood loss 700-1,000 ml. and/or signs of moderate shock

Recovered within one hour

4 cases with moderate shock and minimum blood loss but difficult deliveries

9 cases of blood loss more than $1,000 \mathrm{ml}$. and/or signs of severe shock

6 deaths up to $6 \frac{t}{2}$ hours post partum.

The third patient had a large baby with a labour of 44 hours and a difficult forceps delivery; death was sudden and intra partum. There was no external evidence of haemorrhage, and there was no necropsy.

The fourth patient suffered from essential hypertension; labour was induced by rupture of the membranes followed by spontaneous delivery in 12 hours, and manual removal of a retained placenta was followed by progressive shock and death in three hours. No external bleeding occurred and there was no evidence of ruptured uterus ; no necropsy was done.

The fifth case was one of normal pregnancy and spontaneous delivery. Post-partum haemorrhage was treated by oxytocics (ergometrine and pituitrin) and eventually the uterus was packed.' Progressive shock followed, and the patient died in $6 \frac{1}{2}$ hours despite repeated transfusions. There was no necropsy.

The last case was a normal pregnancy with a breech delivery. There was severe post-partum haemorrhage, treated as in the previous case but followed by death in 5 hours. At necropsy the bleeding was found to have been from the placental site and there was no uterine rupture.

Data of Three Cases of Severe Shock with Recovery.The first of these was a spontaneous delivery with a severe post-partum haemorrhage but with recovery in 12 hours after transfusions. The second was a spontaneous delivery followed by inversion of the uterus and severe post-partum haemorrhage. The patient was transfused and the inversion reduced; recovery occurred in 6 hours. The third was a case of toxic accidental haemorrhage with a spontaneous delivery but severe post-partum haemorrhage. The patient was transfused, and when the haemorrhage persisted hysterectomy was carried out two hours post-partum, being followed by recovery.

\section{Factors in Development of Shock}

During pregnancy there is an increase in the blood volume, which is a favourable circumstance; but with delivery there is an inevitable loss of blood, which may more than overcome the increased volume of pregnancy. The great increase in the size of the uterus, full of large blood sinuses, results in an organ capable of holding a considerable volume of blood when it is relaxed; and its relaxed condition, after being emptied, provides a fertile source of haemorrhage into the uterine cavity.

Disturbance of water balance, which is usually the first indication of toxaemia, is probably evidence of increased permeability of the capillaries, and this, of course, is one of the essential factors in the mechanism of shock. Toxaemic cases with systemic oedema withstand trauma poorly.
The late toxaemias of pregnancy are accompanied by an elevation of blood pressure, which may reach high levels. If convulsions supervene, and even in the absence of eclampsia, . after delivery there is often a very sudden drop in pressure, which is conducive to the onset of shock. During labour the patient is subjected to various types of trauma. To this are often added sleeplessness and apprehension. There is the final mechanical trauma of delivery, which varies from a slight degree of damage to severe injury.

Immediately after delivery there is a marked lowering of intra-abdominal pressure, which probably results in dilatation of the splanchnic blood vessels. This lowering of the intra-abdominal pressure occasionally results in smallbowet distension of such a degree that it may simulate paralytic ileus, and it probably renders the patient more susceptible to shock.

Let us now consider shock in connexion with the various obstetrical accidents.

\section{Shock due to Obstetrical Mishaps}

Haemorrhage.-This, the most important of the obstetrical accidents, may be the result of placenta praevia. The haemorrhage may be slow and prolonged or sudden and rapid, but in either case the blood loss may be sufficient to result in shock. From clinical observation it would appear that a patient suffering from a rapid loss of a considerable amount of blood responds to therapeutic measures better than one who has lost a comparable amount over a longer period of time. In accidental haemorrhage blood loss may be equal to that from severe placenta praevia, but in many instances the shock is out of proportion to the observed blood loss. This is especially true in cases of Couvelaire uterus, or toxic utero-placental apoplexy, in which there is an infiltration of the uterine wall with blood separating the muscle bundles and producing a ligneous, non-contracting uterus. To this is added the fact that practically all of these patients are suffering from a more or less severe toxaemia. In concealed accidental haemorrhage it is difficult to estimate the loss of circulating blood, especially where conservative methods of treatment are the rule, as in our hospital. It may be that the trauma to the uterine musculature is the important factor, but I believe that blood loss is more important. And, finally, there is post-partum haemorrhage of two types-haemorrhage following trauma with tearing of the birth canal, and bleeding due to failure of an atonic uterus to contract and shut off the blood sinuses. In most instances the haemorrhage is external and therefore apparent, but occasionally when it results from injury 'to the birth canal it may be entirely within the pelvis as a retroperitoneal haematoma, or occasionally as an intraperitoneal haemorrhage.

Rupture of the Uterus.-This mishap is probably more common than is recognized clinically. It is most common through the scar of a previous section, next after difficult operative deliveries; but it sometimes occurs spontaneously even in unobstructed labours. It would appear that rupture of the uterus per se seldom produces clinical evidence of shock, and when the latter develops it is secondary to haemorrhage. In Eastman's series shock was present in less than half the cases of scar rupture, and in one-third of the cases of spontaneous rupture vaginal delivery was carried out without realizing that rupture had occurred. I have seen three cases of complete and one of incomplete rupture with no evidence of shock. It is obvious that the bleeding must have been slight in these instances.

Inversion of the Uterus.-Inversion may occur spontaneously or be due to unskilful management of the third stage of labour. A considerable number of these cases 
show shock quickly. A large proportion of the remainder will subsequently go into shock either as the result of haemorrhage or from attempts at replacement, which are frequently accompanied by bleeding, often profuse. All cases of puerperal inversion of the uterus should therefore be looked upon as potential cases of shock, and prophylactic measures against this condition should be at-once instituted, before any attempt at its correction is carried out. Nevertheless, all cases of acute inversion do not show shock. During the past ten years we have seen seven cases of acute inversion: one had profuse bleeding with severe shock; two had moderate haemorrhage and moderate shock; the other four cases had no evidence of shock.

Eclamptic Toxaemia.-Hailey, Schwartz, and Adair, as well as other observers, have pointed out that marked vasomotor collapse is sometimes the cause of death in eclampsia, and even in pre-eclampsia. Hunt and Adair collected eight cases in which the systolic pressure dropped by 100 to $200 \mathrm{~mm}$. within a few minutes, and all of these cases showed clinical evidence of shock. Rapid delivery or deep anaesthesia may aggravate a potential case. On the other hand, it is known that hypertension cases of both sexes stand operation well. The collapse that follows delivery in cases of hypertension is probably explained by a sudden lowering of the intra-abdominal pressure with a filling of the splanchnic vessels with non-circulating blood. When death does not result quickly from the shock it may occur later from damage to the central nervous system due to cerebral anaemia. MacGlamman has pointed out that during caesarean section on non-eclamptic cases there is no marked drop in blood pressure ; whereas if the section was done in the case of eclampsia the fall in pressure as the uterus was emptied was frequently great-in one case $90 \mathrm{~mm}$. in a period of 40 minutes.

Psychic causes during labour occasionally result in syncope. It is a debatable question whether such causes may produce true shock. Some observers believe that it does, and Miles Phillips thinks that emotional factors are important in the production of shock, and quotes a fatal case of Whitridge Williams's. DeLee also was of the opinion that this was sometimes a factor of importance. In Crile's theory of shock the aetiology was thought to be a combination of fear and anxiety with pain, leading to a state of mental and physical exhaustion, and eventually to shock. In any event, fatigue and starvation after prolonged labour should not be labelled "shock."

\section{Management of Obstetric Shock}

It is much easier to prevent the onset of shock than it is to treat the condition after it has become established. If the obstetrician has constantly in mind those conditions leading to shock, most particularly haemorrhage, he will make every effort to obviate them and to provide for their treatment before constitutional symptoms have made their appearance. In the case of haemorrhage the blood loss should be replaced as rapidly as possible, but of more importance is to try to prevent any undue bleeding. In cases suffering from hypertension delivery should never be rapid, and after delivery, if there is any marked drop in blood pressure, a tight abdominal binder should be applied, and to this may be added the weight of a sandbag. Mechanical trauma should be kept to a minimum, and when it occurs should be recognized and treated. As I have already pointed out, in all cases of inversion of the uterus anti-shock therapy should be instituted at once, even if shock has not yet developed.

In the active treatment of shock the first requisite is the abolition of pain and apprehension by the administration of morphine. Means should be taken to retain the body heat. A temperature of 90 to $100^{\circ}$ surrounding the body should not cause damage. In our hospital such heat is supplied in the form of warm air, thermostatically controlled by an electric heater, and no heated bottles or pads are placed in the patient's bed. The replacement of the circulating blood volume, however, is the prime essential, and any loss of time in this procedure should be avoided. In the larger hospitals to-day blood banks are usually in operation, and therefore a transfusion of blood or plasma should be available within ten minutes. Blood banks are not feasible in most of the smaller hospitals, and when these are situated at some distance from a blood bank a supply of plasma should always be on hand; to-day this is easily arranged. In cases without severe haemorrhage the plasma is of equal value with whole blood, and even when bleeding is profuse plasma is of great service. When delivery has occurred in a home, and particularly in rural districts, the obstetrician should always have facilities for the intravenous administration of glucose solution and should use it early.

Lastly, where shock has made its appearance, the trauma of any operative procedure should be avoided except where such procedure is necessary to stop active haemorrhage. In placenta praevia measures to stop the haemorrhage should be carried out while the replacement of the lost blood is going on. Indeed, when operative measures for placenta praevia are contemplated, even where there has as yet been no great blood loss, a transfusion prior to or during operation is advisable to forestall possible haemorrhage during operation. In cases of accidental haemorrhage where shock does not exist I believe a conservative method of treatment is most apt to avoid its development. In most cases of Couvelaire uterus, with the patient already in shock, the active haemorrhage has usually ceased, and any blood that appears at the vulva is that which has already poured out into the interior of the uterus. The prime requisite, therefore, is to treat the shock and not to add to it by the trauma of operative delivery: If the patient does not respond to morphine, transfusion, and heat, operation would not have saved her life; if she does respond to such measures one usually finds that the uterus has regained its contractile powers and delivery proceeds normally. Our treatment of accidental haemorrhage is conservative. Where shock does not exist there is no question in my mind that this is emphatically the best attitude. In cases of toxic accidental haemorrhage it is usually the method of choice. There may be a place for caesarean section in the treatment of toxic accidental haemorrhage, but I believe the place is small. Where severe shock does not exist and the baby is still alive and viable section may be indicated in the interest of the child, and in a few cases where fresh haemorrhage continues to occur and labour does not supervene it may be indicated after the shock has been treated.

\section{Summary of Conclusions}

From the clinical point of view the great majority of cases of obstetric shock are directly proportional to the amount of blood loss. This loss may be apparent or it may be concealed.

When confronted with a case of obstetric shock without obvious cause the history should be meticulously reviewed from the standpoint of possible causes of concealed bleeding, especially rupture of the uterus.

The prevention of haemorrhage is of first importance, but the replacement of lost blood is the sine qua non of treatment. Where haemorrhage is anticipated transfusion should be started before operative procedures are carried out.

With the first evidence of shock treatment should be started promptly before the condition has become irreversible.

In obstetric hospitals where blood banks have not yet been estahlished supp ies of plasma should be available at all times. 